\title{
Dynamic Channel Allocation for effective self Reconfigurable wireless mesh network
}

\author{
Avinash Devare ${ }^{1}$, R.V.Mulajkar ${ }^{2}$, \\ '(ME student SCOE Vadgone, Pune) ${ }^{2}$ (ME Student GECA Aurangabad)
}

\begin{abstract}
Wireless Mesh Networks (WMNs) consist of mesh routers and mesh clients for their lifetime, multihop wireless mesh networks (WMNs) experience frequent link failures caused by channel interference, dynamic obstacles, and/or applications' bandwidth demands. Dynamic channel allocation for effective autonomous network reconfiguration system (E-ARS), by analyzing E-ARS, it shows that by using EARS alone it won't provide a sufficient result such as network quality, leader assigning problems etc, so in order improve the network performance we going to implement a new concept Breadth First Search Channel Assignment (BFS-CA) algorithm against with E-ARS so that it will multi radio configuration for mesh network and channel assignment problems. We demonstrate our solution's through the evaluation of a prototype implementation in an IEEE 802.11 in ns2. We also report on an extensive evaluation via simulations. In a sample multi-radio scenario, our solution yields performance more gains compared E-ARS.
\end{abstract}

Keywords: IEEE 802.11, multiradio wireless mesh networks (mr-WMNs), E-ARS, BFS-CA networks, wireless link failures.

\section{INTRODUCTION}

Wireless Mesh Network is growing very widely now a day. It is used in various applications such as public safety. Even though many solutions for WMNs to recover from wireless link failures have been proposed, they still have limitations as follows. First Autonomous reconfigurable system (ARS) outperforms existing failure-recovery methods, such as static greedy channel assignments, and local rerouting. Second load on channel vary over time. To achieve our goals then the existing work E-ARS, we propose, a new concept Breadth First Search Channel Assignment (BFS-CA) algorithm against with E-ARS so that it will multi radio configuration for mesh network and channel assignment problems. To sense and evaluate the current channel conditions, our network includes dynamic channel sensor nodes for multi-radio dynamic configuration. The dynamic channel detection sensors monitor and record the traffic on each channel. Using this technique BFSARS network with E-ARS, we show the performance of some experiments to determine the best set of properties about each different channel to be able to rank all available channels in terms of achievable performance.

We can then build a dynamic channel allocation using ARS algorithm that will, according to the appropriate set of properties about the current channel utilization, choose and dynamically assign the best channel for the links in our wireless mesh network. A comprehensive performance study that shows significant throughput improvements in the presence of varying interference levels, which are validated through empirical measurements on a prototype implementation.

Wireless mesh network consist of mesh router, mesh client, gateway. Functionality of mesh router is to forward packet (traffic) to and from gateway which is connected to internet. Mesh client is nothing but your laptop or mobile. Gateway is one of entry or exit point from one network to another.

In section I we define motivation for our project and II drawbacks of existing system. Section III explains scope of our project. In section IV we have explained Literature review, architecture of system and finally conclusion.

\section{MOTIVATION}

The channel assignment algorithm we propose in this paper is designed for wireless mesh networks. Routers in such networks are stationary. Whereas, user devices, such as laptops and PDAs, can be mobile. Such devices associate with routers. In ARS there is a leader node which is chosen by group member so whenever link failure occurs that information is given to the leader node. Leader node forward that information to gateway and all the functionality (like routing planning, reconfiguration) performed at gateway and send back to leader node. Finally, all nodes in the group execute the corresponding configuration changes.

The main drawback of dynamic channel assignment is that it results into change in network topology, so to avoid this solution is that make mandatory one radio of mesh router to operate on default channel. This default radio is of the same physical layer technology IEEE $802.11 \mathrm{a}, 802.11 \mathrm{~b}$ or $802.11 \mathrm{~g}$. A second drawback 
is channel assignment can result in disruption of flows when the mesh radios are reconfigured to different frequencies. To prevent flow disruption, redirect flow over default channel.

Channel Assignment (CA) in a multi radio WMN environment consists of assigning channels to the radio interfaces in order to achieve efficient channel utilization and minimize interference. we describe different schemes that can be used to assign channels in a wireless mesh network. These schemes are generally classified as: Static, Dynamic and Hybrid Channel Assignment.

III. COMPARISION WITH EXISTING ALGORITHM

\begin{tabular}{|c|c|c|c|c|c|c|}
\hline & Property & Switching Time & Connectivity & Ripple Effect & Topology control & $\begin{array}{c}\text { Control } \\
\text { philoso } \\
\text { phy }\end{array}$ \\
\hline \multirow{6}{*}{$\begin{array}{l}\text { FIXED } \\
\text { CA }\end{array}$} & $\mathrm{CCA}$ & No & $\begin{array}{c}\text { Ensured by CA } \\
\text { scheme }\end{array}$ & No & Fixed & $\mathrm{NA}$ \\
\hline & C-HYA & No & $\begin{array}{c}\text { Ensured by CA } \\
\text { scheme }\end{array}$ & Yes & Fixed & $\mathrm{CS}$ \\
\hline & CLICA & NO & $\begin{array}{c}\text { Ensured by CA } \\
\text { scheme }\end{array}$ & No & $\begin{array}{c}\text { CA scheme define } \\
\text { topology }\end{array}$ & $\mathrm{CS}$ \\
\hline & MICA & No & $\begin{array}{c}\text { Ensured by CA } \\
\text { scheme }\end{array}$ & No & $\begin{array}{c}\text { CA scheme define } \\
\text { topology }\end{array}$ & $\mathrm{CS} / \mathrm{DS}$ \\
\hline & MesTiC & No & $\begin{array}{c}\text { Ensured by CA } \\
\text { scheme }\end{array}$ & No & Fixed & $\mathrm{CS}$ \\
\hline & TiMeSH & No & $\begin{array}{c}\text { Ensured by CA } \\
\text { scheme }\end{array}$ & No & $\begin{array}{c}\text { CA scheme define } \\
\text { topology }\end{array}$ & DS \\
\hline \multirow{2}{*}{$\begin{array}{c}\text { HYBRI } \\
\text { D } \\
\text { CA }\end{array}$} & LLP & YES & $\begin{array}{c}\text { Ensured by } \\
\text { channel switching }\end{array}$ & No & $\begin{array}{c}\text { Dynamically } \\
\text { changing }\end{array}$ & DS \\
\hline & BFS-CA & $\begin{array}{c}\text { YES } \\
\text { (INFREQUENT) }\end{array}$ & $\begin{array}{c}\text { Ensured by default } \\
\text { radio }\end{array}$ & No & Fixed & $\mathrm{CS}$ \\
\hline $\begin{array}{l}\text { DYNA } \\
\text { MIC } \\
\text { CA }\end{array}$ & D-HYA & $\begin{array}{c}\text { YES } \\
\text { (INFREQUENT) }\end{array}$ & $\begin{array}{c}\text { Ensured by CA } \\
\text { scheme }\end{array}$ & No & $\begin{array}{c}\text { No topology is } \\
\text { defined by routing } \\
\text { tree }\end{array}$ & DS \\
\hline
\end{tabular}

* CS=centralized System DS=Distributed System

\section{SCOPE}

ARS is used for static channel allocation so we are developing a system to overcome this limitation named Dynamic channel allocation for effective autonomous network reconfiguration system (E-ARS). We propose, and evaluate a wireless mesh network with dynamically allocated channels. To sense and evaluate the current channel conditions, our network includes dynamic channel sensor nodes. The channel detection sensors monitor and record the traffic on each channel. Using this network, we can first perform some experiments to determine the best set of properties about each different channel to be able to rank all available channels in terms of achievable performance. We can then build a dynamic channel allocation using ARS algorithm that will, according to the appropriate set of properties about the current channel utilization, choose and dynamically assign the best channel for the links in our wireless mesh network. We can then evaluate the performance gains offered by this allocation engine.

The explosive growth in Wi-Fi deployments that operate in the same spectrum as wireless mesh networks, any static assignment will likely result in the operation of the mesh on channels that are also used by co-located Wi-Fi deployments. The resulting increase in interference can degrade the performance of the mesh network. For this reason our Channel Assignment algorithm addresses the channel assignment problem and specifically investigates the dynamic assignment of channels in a wireless mesh network. We chose the hybrid and centralized, interference-aware channel assignment algorithm BSF-CA as this channel assignment protocol aimed at improving the capacity of wireless mesh networks by making use of all available non-overlapping channels (i.e. IEEE 802.11) and that intelligently selects channels for the mesh radios in order to minimize interference within the mesh network and between the mesh network and co-located wireless networks. Hence, our first improvement will try to introduce this new information into the algorithm. Second, we will develop an algorithm that can be used in a wireless mesh network with more gateways available, this because the BSF-CA algorithm is designed to work on single-gateway WMNs. 


\section{LITERATURE REVIEW}

With ever-increasing demands for pervasive access to the Internet, multi-hop wireless networks have been actively developed and widely deployed. A recent study has shown that more than cities and counties in the U.S. have been deploying multi-hop or large-scale wireless networks. In addition, these multi-hop networks have created new applications and services, including municipal broadband Internet, emergency response systems, and climate/environment monitoring systems. By using inexpensive wireless links such as IEEE 802.11, multi-hop networks can easily provide network connectivity over target areas without deploying expensive wire-lines. Furthermore, because of their multi-hop nature, these networks can significantly improve spectrum utilization and network reliability by increasing spatial spectrum reuse as well as redundancy in network topology (e.g., mesh), compared to traditional cellular networks. Before designing ARS, it was necessary to understand the need of Self- reconfiguration

\section{- Recovering from link-quality degradation:}

The quality of wireless links in WMNs can degrade (i.e., link-quality failure), due to severe interference from other co-located wireless networks. For example, Bluetooth, cordless phones, and co-existing wireless networks operating on the same or adjacent channels cause significant and varying degree of losses or collisions in packet transmissions, as shown in By switching the tuned channel of a link to other interferencefree channels, local links can recover from such a link failure.

\section{- Satisfying varying QoS demands:}

Links in some areas may not be able to accommodate increasing QoS demands from end users (QoS failures), depending on spatial or temporal locality. For example, links around a conference room may relay too much data/video traffic during the session. Likewise, relay links outside the room may fail to meet requests of attendees' Voice-over-IP calls during a session break. By re associating their radios/channels with under-utilized radios/channels available nearby, links can avoid the failures.

\section{- Coping with heterogeneous channel availability:}

Links in some areas may not be able to access certain wireless channels during a time period (spectrum failures), due to spectrum etiquette or regulation. For example, some links in a WMN need to vacate current channels if channels are being used for emergency response near the wireless links (e.g., hospital, public safety). Such links can seek and identify alternative channels available in that area. Motivated by these three and other possible benefits of using reconfigurable mr-WMNs, in the remaining, we would like to develop a system that allows mr-WMNs to autonomously change channel and radio.

\section{ARCHITECTURE OF SYSTEM}

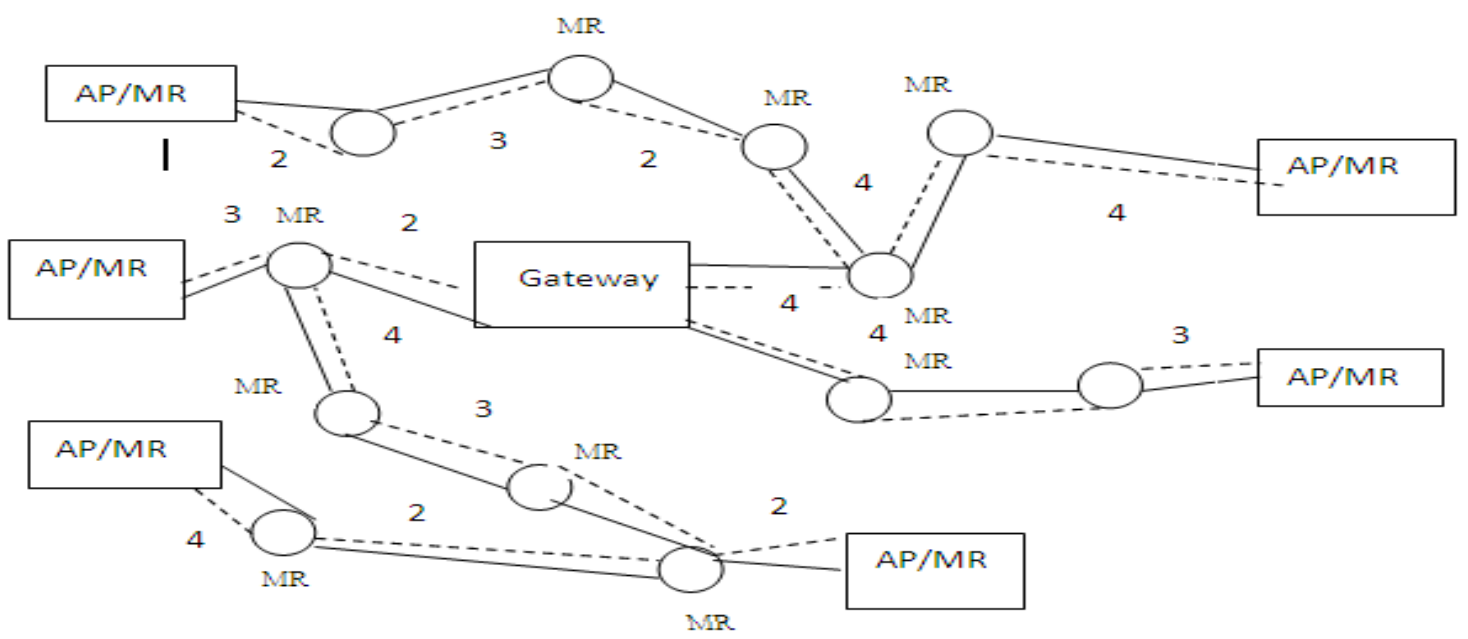

fig Architecture of E-ARS.

In this fig Dotted link shows non-overlapping channel. Solid line shows overlapping channel. Fig. shows multiradio multichannel wireless mesh network. The mesh routers are assumed to be equipped with multiple IEEE 802.11 radios, such as $802.11 \mathrm{a}, 802.11 \mathrm{~b}$, or $802.11 \mathrm{~g}$. Here we need to mandate each MR will be associate with one radio, called the default radio, which is of the same physical layer type, e.g. 802.11b. One of the routers is assigned as gateway or Channel Allocation Server (CAS). Access Points (APs) provide connectivity to user 
devices and are co-located with mesh routers. A majority of the traffic within the mesh is either from the user devices to the gateway or vice-versa. Therefore, in order to improve overall network capacity, it is preferable to place MRs close to the gateway and in regions of the mesh that are likely to experience heavy utilization.

The dotted lines in the figure show links between MRs that are tuned to non-overlapping channels. In our example, five such channels are used. A sixth channel, indicated by solid lines, is the default channel. The Channel Assignment Server (CAS), which is co-located with the gateway in the figure, performs channel assignment to radios.

\section{E-ARS Algorithm}

\section{ALGORITHM}

Algorithm 1: AS Operation at mesh node

Step 1: Generate topology and construct MCG

Step 2: Start flooding information

A: for every link/node do

B: Exchange neighbor Nodes information.

C: end for

D: send neighbor node information to the gateway or CAS

Step 3: Select source node and Destination node As well as assign default and non default channel

Step 4: Establish path from source to destination

Step 5: Start packet transmission.

Step6: Check node/link failures else go to step 10

Step 7: if link failure occurs in same group

a) Use bully algorithm

b) Choose leader node amongst them.

c) Start reconfiguration and

d) Generate Reconfigure plan.

e) Re-establish path.

f) send information to gateway (CAS)

Step 9: Start packet transmission on default channel.

Step 10: Receive packets

Step 11: Stop

The algorithm starts by adding all vertices from the MCG to a list, $V$. It does a breadth first search of the MCG to visit all vertices and assign them channels. The search starts from vertices that correspond to links emanating from the gateway the smallest hop count vertex is determined of all vertices in the MCG. All vertices with distance equal to the smallest hop count are added to a queue, $Q$. If vertices correspond to network links emanating from the gateway, their hop count is 0.5 . These vertices are then sorted by increasing delay values This sort is performed in order to give higher priority to the better links emanating from the shortest hop count router (the gateway for the first BFS iteration). The algorithm then visits each vertex in $Q$ and permanently assigns them the highest ranked channel that does not conflict with the channel assignments of its neighbors If a non-conflicting channel is not available, a randomly chosen channel is permanently assigned to the vertex. Note, however, that the default channel is never assigned. Once a vertex is assigned a channel, all vertices that contain either radio from the just-assigned vertex are placed in a list, $L$. All vertices from $L$ are removed from the MCG. This step is needed to satisfy the constraint that only one channel is assigned to each radio. The radios in the list of vertices that do not belong to the just-assigned vertex are tentatively assigned the latter's channel

Vertices at the next level of the breadth first search are added to $Q$. These vertices correspond to links that fan-out from the gateway towards the periphery. To find such links in the MCG, two steps are performed. In the first step, the router from the just-assigned vertex that is farthest away from the gateway is chosen; the farthest router is the router with the higher hop-count of the two routers that make up the just-assigned vertex. In the second step, all unvisited MCG vertices that contain a radio belonging to the farthest router are added to the list, Tail. This list is sorted by increasing value of the delay metric to give higher priority to better links that emanate from the farthest router. Finally, the vertices from Tail are added to $Q$. The above described algorithm continues until all vertices in the MCG are visited. If there is a link failure while transmitting a packet do use of bully algorithm and on that basis select leader node amongst them. Start reconfiguration and generate reconfiguration plan. Reestablish path. Any radio that is not assigned a permanent channel during the search, because vertices containing it were deleted, is permanently assigned one of the channels tentatively assigned to it. Once channel assignments are decided, the CAS notifies the mesh routers to re-assign their radios to the chosen channels. 


\section{CONCLUSION}

Dynamic channel allocation for effective autonomous network reconfiguration system (E-ARS), by analysing E-ARS, it shows that by using E-ARS alone it won't provide a sufficient result such as network quality, leader assigning problems etc, so in order improve the network performance we going to implement a new concept Breadth First Search Channel Assignment (BFS-CA) algorithm against with E-ARS so that it will multi radio configuration for mesh network and channel assignment problems.

\section{ACKNOWLEDGMENT}

I would like to express my sincere gratitude towards my parents, my family and friends, for always being there with me With all respect and gratitude, I would like to thank all the people, who have helped me directly or indirectly. Without their silent support and encouragement for this work could not have been possible. I would wish to thank Prof M.P.Wankhade and Prof S.G.Shikalpure for his valuable and firm suggestion, guidance and constant support throughout his work.

\section{REFERENCES}

[1]. I. Akyildiz, X. Wang, and W. Wang, "Wireless mesh networks: A survey," Comput. Netw., vol. 47, no. 4, pp. 445-487, Mar. 2005.

[2]. K. Ramanchandran, E. Belding-Royer, and M. Buddhikot, "Interference- aware channel assignment in multi-radio wireless mesh networks," in Proc. IEEE INFOCOM, Barcelona, Spain, Apr. 2006.

[3]. M. Alicherry, R. Bhatia, and L. Li, "Joint channel assignment and routing for throughput optimization in multi-radio wireless mesh networks," in Proc. ACM MobiCom, Cologne, Germany, Aug. 2005

[4]. A. P. Subramanian, H. Gupta, S. R. Das, and J. Cao, "Minimum interference channel assignment in multiradio wireless mesh networks,"IEEE Trans. Mobile Comput., vol. 7, no. 12, pp. 1459-1473, Dec. 2008

[5]. K.-H. Kim and K. G. Shin, "On accurate and asymmetry-aware measurement of link quality in wireless mesh networks," IEEE/ACMTrans.Netw., vol. 17, no. 4, pp. 1172-1185, Aug. 2009.

[6]. P. Kyasanur and N. Vaidya, "Capacity of multi-channel wireless networks:Impact of number of channels and interfaces," in Proc. ACM MobiCom, Cologne, Germany, Aug. 2005, pp. 43-57.

[7]. A. Brzezinski, G. Zussman, and E. Modiano, "Enabling distributed throughput maximization in wireless mesh networks: A partitioning approach," in Proc. ACM MobiCom, Los Angeles, CA, Sep. 2006, pp.2637.

[8]. S. Chen and K. Nahrstedt, "Distributed quality-of-service routing in ad hoc networks," IEEE J. Sel. Areas Commun., vol. 17, no. 8, pp.1488-1505, Aug. 1999

[9]. P. Bahl, R. Chandra, and J. Dunagan. SSCH: Slotted Seeded Channel Hopping For Capacity Improvement in IEEE 802.11 Ad Hoc Wireless Networks. In ACM MobiCom, Philadelphia, PA, September 2004.

[10]. R. Draves, J. Padhye, and B. Zill. Routing in Multi-radio, Multihop Wireless Mesh Networks. In ACM MobiCom, Philadelphia, PA, September 2004.

[11]. P. Gupta and P. Kumar. Capacity of Wireless Networks. In IEEE Transactions on Information Theory, volume 46, pages 388-404, March2000. 